\title{
Image Enhancement of Transformer Oil Images Using Improved Complex Shock Filter
}

\author{
Chaldiganahalli Mallappa Maheshan ${ }^{1,2 *}$, Hombalaiah Prasanna Kumar ${ }^{2}$ \\ ${ }^{1}$ Department of Electrical Engineering, Visvesvaraya Technological University-Research Resource Centre (VTU-RRC), Jnana \\ Sangama, Belagavi 590018, Karnataka State, India \\ ${ }^{2}$ Department of Electrical Engineering, University Visvesvaraya College of Engineering (UVCE), Bangalore University, \\ K.R.Circle, Bengaluru 560001, Karnataka, India
}

Corresponding Author Email: maheshan.cm@gmail.com

https://doi.org/10.18280/i2m.180410

Received: 12 April 2019

Accepted: 20 July 2019

\section{Keywords:}

CSF, ICSF, MSE, OSF, PSNR, SSIM,

transformer oil

\begin{abstract}
This paper aims to propose a novel improved complex shock filter for image enhancement of real time transformer oil images. An improved theoretical equation was introduced for the analysis in order to normalize the complex diffusion shock filter. This was accomplished through multiplying each term of complex shock filter by scalar correction constant to crumbling into three cases of improved complex shock filter. The results of all the cases are validated using image performance metrics such as Mean Square Error (MSE), Peak Signal to Noise Ratio (PSNR) and Similar Structure Index Mean (SSIM).Through this study, it was found that the proposed noise elimination method can be effectively used for synthetic as well as real time transformer oil images without affecting the structure of the object. The findings of this research may serve as a new filtering technique for the analysis of an image.
\end{abstract}

\section{INTRODUCTION}

Transformer is a static device used transfer energy from one voltage level to other with a constant frequency and power. Performance of this continuously working equipment is based on the insulation and cooling characteristics. In general, mineral oil from crude petroleum used as dielectric as well as cooling medium in all the power transformers [1-3]. Gases produced and dissolved in the transformer oil due to operation of transformer at various temperatures for different loads minimize the cooling as well as insulation efficiency. Hence transformer oil chemical, structural and thermal characteristics are to be monitored to improve the effectiveness performance of the transformer [4-6]. There are many time-consuming classical methods are available to measure the performance of transformer oil. The digital image processing is an emerging non-destructive method for diagnosis of internal faults occurred transformer [7, 8].

A picture with two dimensions representing individual/distinct features such as structure, shape, texture and colour etc. is termed as an image [9-11]. Digital images of transformer oil captured through digital camera are associated with different noise due to inadequate illumination levels as well as natural or forced convection movement of oil. There are numerous linear and nonlinear filters exists in the literature for denoising and edge preservation of the images. Shock filter is best suited for transformer oil images since it operates at different temperatures in addition to abrupt variation of temperature of oil. Firstly, the shock filter proposed by Kramer and Bruckner [12] based on dilation process near a maximum and erosion near minimum. The introduction of original shock filter in [13] is very sensible and cannot remove noise. Many authors proposed diffusion schemes in shock filter such as smoothed Laplacian [14], while Gilboa, et al. [15] normalize the shock in complex domain.

In this paper a novel improved complex shock filter is proposed and tested its performance with the synthetic in addition to real transformer oil images. The objective of this paper to enhance the transformer oil images captured at different temperatures through improved complex shock filter. The visual and numerical results of the proposed method is compared with other image filtering enhancement techniques such as median, wiener, shock along with complex shock filter. Numerical results are validated through image quality evaluation metrics such as MSE, PSNR and SSIM [16-18].

This paper is organized as follows: section 2 introduces the shock filter along with complex diffusion shock filter, section 3 describes the proposed method, section 4 furnishes the comprehensive experimental results and discussions, and in section 5 the conclusion of the paper presented.

\section{SHOCK FILTER}

Original Shock Filters (OSF) are morphological image enhancement techniques which processes each pixel of an image using Partial Differential Equations (PDE). The term shock filtering introduced by Osher and Rudin [13] based on hyperbolic PDE. In general, 1D (one-dimensional) shock filter described by PDEs:

$$
\mathrm{I}_{\mathrm{t}}=\frac{\partial I}{\partial t}=|I x| \mathrm{F}(I x x)
$$

where, I represent the image. I $x$ and I $x x$ represent first and the second directional derivatives of the image I respectively. Function $\mathrm{F}$ must satisfy $\mathrm{F}(0)=0, \mathrm{~F}(\mathrm{~s}) \operatorname{sign}(\mathrm{s}) \geq 0$. Equation (1) satisfies Neumann boundary conditions with initial conditions $\mathrm{I}(\mathrm{x}, 0)=\mathrm{I}_{0}(\mathrm{x})$. Choosing $\mathrm{F}(\mathrm{s})=\operatorname{sign}(\mathrm{s})$ gives the 
shock filter equation in $1 \mathrm{D}$ as:

$$
\mathrm{I}_{\mathrm{t}}=-\operatorname{sign}(\mathrm{I} x x)|\mathrm{I} x|
$$

In the 2D (two-dimensional) case the shock filter equation is commonly generalized to:

$$
\mathrm{I}_{\mathrm{t}}=-\operatorname{sign}(\mathrm{I} \eta \eta)|\nabla \mathrm{I}|
$$

where, $\eta$ is direction of gradient $|\nabla \mathrm{I}|$.

OSF used in image processing enhancement to remove blurs edges in the image. But they are exceptionally sensitive to noise signal that is any noise in the blurred signal will be enhanced. A blur edge steep input signal and its OSF output is as shown in Figure 1(a) - 1(b). Similarly, the noisy edge input signal along with OSF output is characterized in Figure $1(\mathrm{c})-1(\mathrm{~d})$ respectively. The output of OSF is not completely enhanced with noisy steep blur edge input signal but when the input signal is steep blur edge input an unambiguously enhancement can be observed Figure 1(a) - 1(d).

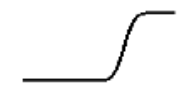

(a)

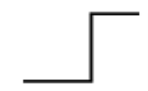

(b)

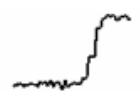

(c)

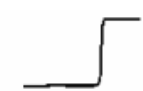

(d)
Figure 1. (a) Blur edge input signal b) OSF output for blur edge input signal (c) Noisy edge input signal (d) OSF output for noisy edge input signal

The noise in the blurred input signal is not completely removed using OSF. The advance in formulation of PDEs is developed to eliminate the difficulties in classical shock filter. Gilboa et al. [15] combined shock and diffusion in their work to form complex diffusion shock filter (CSF). The PDE equation for $2 \mathrm{D}$ image with nonlinear complex diffusion approach has of the form

$$
\mathrm{I}_{\mathrm{t}}=-\frac{2}{\pi} \arctan \left(\mathrm{a} \operatorname{Im}\left(\frac{I}{\theta}\right)\right)|\nabla \mathrm{I}|+\lambda \operatorname{I\eta } \eta+\tilde{\lambda} \operatorname{I} \varepsilon \varepsilon
$$

where, $\lambda=\mathrm{re}^{\mathrm{i} \theta}$ is a complex scalar, $\tilde{\lambda}$ is a real scalar, $\theta \in\left(-\frac{\pi}{2}, \frac{\pi}{2}\right)$ is the phase angle, Im represents imaginary and parameter a control the sharpness of the slope near zero. The gradient norm $|\nabla \mathrm{I}|$ in equation (3) and (4) is computed using a slope limiter minmod function in order to minimize the sudden signal variations [19].

\section{PROPOSED METHOD}

The transformer oil subjected to variation in temperature during the operation of in service transformer. Image captured at different temperatures are enhanced using different filters. Since the removal of undesirable information is not completely achieved by Classical filters as well as the OSF or CSF, the novel Improved CSF is proposed in this paper to enhance the digital transformer oil image obtained at different temperature. The CSF defined in eq. 4 is modified for the theoretical analysis by multiplying each term of this equation with real scalar correction constant $\mathrm{C}$. The performance of this novel Improved Complex Shock Filter (ICSF) are validated by comparing the results obtained by ICSF with the results of median, wiener, OSF and CSF. Eq. (4) develop into three different cases as:
Case 1:

$$
\mathrm{I}_{\mathrm{t}}=-\frac{2}{\pi} \arctan \left(\operatorname{aIm}\left(\frac{I}{\theta}\right)\right)|\nabla \mathrm{I}|+\lambda \mathrm{I} \eta \eta+C(\tilde{\lambda} \mathrm{I} \varepsilon \varepsilon)
$$

Case 2:

$$
\mathrm{I}_{\mathrm{t}}=-\frac{2}{\pi} \arctan \left(\operatorname{aIm}\left(\frac{I}{\theta}\right)\right)|\nabla \mathrm{I}|+C(\lambda \mathrm{I} \eta \eta)+\tilde{\lambda} \mathrm{I} \varepsilon \varepsilon
$$

Case 3:

$$
\mathrm{I}_{\mathrm{t}}=C\left(-\frac{2}{\pi} \arctan \left(\operatorname{aIm}\left(\frac{I}{\theta}\right)\right)|\nabla \mathrm{I}|\right)+\lambda \operatorname{I\eta } \eta+\tilde{\lambda} \mathrm{I} \varepsilon \varepsilon
$$

All three cases of the filter possess new variable $\mathrm{C}$ in addition to the all the variables of Eq. 4. In case 1, the quantity of diffusion in intensity set direction altered by multiplying $\mathrm{C}$ with the last term having $\tilde{\lambda} \mathrm{I} \varepsilon \varepsilon$. In case $2, \mathrm{C}$ is multiplied with the middle term containing $\lambda$ I $\eta \eta$ to adjust the complex diffusion term intended for removal of noise. In case 3 , the false inflection points produced by noise are accustomed by multiplying $\mathrm{C}$ with first term having trigonometric tan function. The results of each case tabulated in table 1 for the values of $\mathrm{C}=0.2,0.4,0.6$ and 0.8 . Figure 2 describes the flowchart of proposed method.

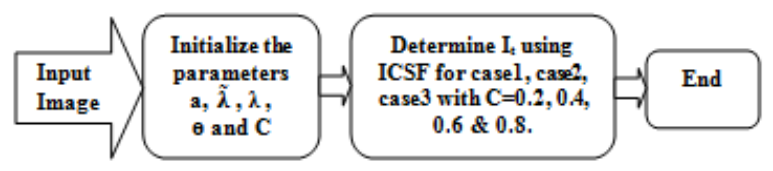

Figure 2. Flow chart of proposed ICSF

\section{EXPERIMENTAL RESULTS AND DISCUSSIONS}

In this section, the experimental results of proposed method on both synthetic images and real transformer oil images, and compare experimental results of proposed method with other image enhancement methods. The performance of proposed method along with other image enhancement filtering techniques is evaluated using image quality metrics such as MSE, PSNR and SSIM.

\subsection{Performance on synthetic images}

In this part, the visual plus numerical results obtained on three classical synthetic images: Lena, baboon, peppers are established. Figure 3 presents the gray image, the denoised images obtained in all three cases of proposed ICSF method with real scalar correction constant $\mathrm{C}=0.2$ in addition to other filtering methods like shock as well as complex shock filter. Further to enumerate the performance, the results obtained with proposed ICSF are compared with conventional median and wiener filtering methods in addition to OSF as well as CSF. The filtered images obtain with ICSF method with case $2 / \mathrm{C}=0.2$ appear to be smoothed with a better edge and shape preservation compared with the other methods. Figure 4 shows the gray image, resultant synthetic images obtained from median, wiener, OSF, $\mathrm{CSF}$ and $\mathrm{ICSF} /$ case $2 / \mathrm{C}=0.2$ filtering methods. To quantify the denoising qualities, first three columns of Table 1 present the numerical results for classical synthetic images. The performance criterion used is MSE, PSNR and SSIM. It can be observed that the proposed 
ICSF method has low MSE, high PSNR \& on the brink of unity SSIM.

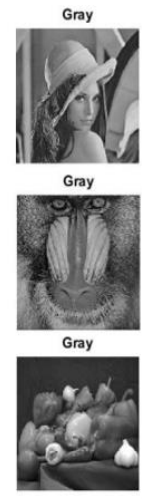

(a)

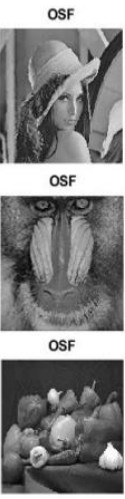

(b)

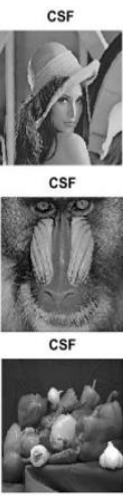

(c)

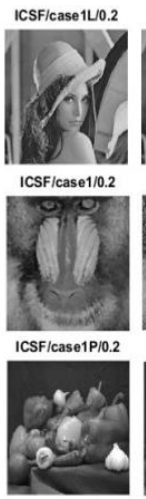

(d)

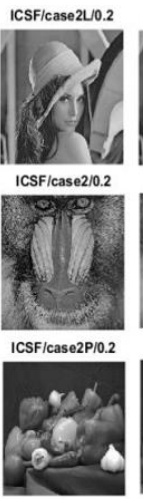

(e)

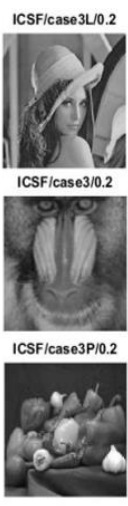

(f)
Figure 3. Top to bottom gray images of Lena, baboon and peppers. Enhanced images using (b) OSF (c) CSF (d) $\mathrm{ICSF} / \mathrm{CASE} 1 / \mathrm{C}=0.2$, (e) ICSF/CASE2/C $=0.2$, and (f) $\mathrm{ICSF} / \mathrm{CASE} 2 / \mathrm{C}=0.2$

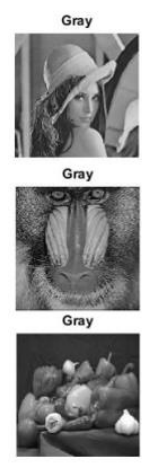

(a)
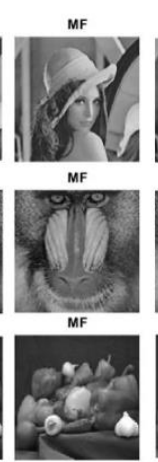

(b)

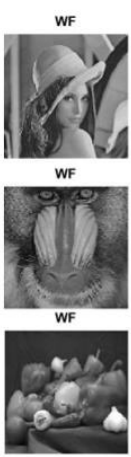

(c)

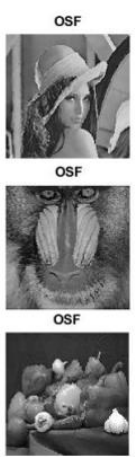

(d)

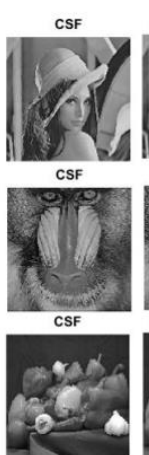

(e)

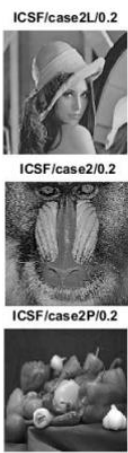

(f)
Figure 4. Top to bottom gray images of Lena, baboon and peppers. Enhanced images using (b) Median Filter, (c)wiener filter, (d) OSF, (e) CSF and (f) ICSF / CASE2 / C =0.2

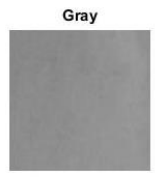

ICSF/case $1 T / 0.6$

ICSF/case 2 T/0.8

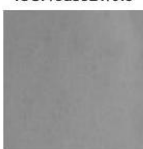

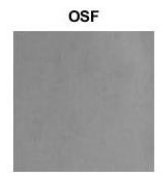

ICSF/case $1 T / 0.8$
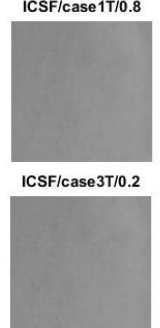

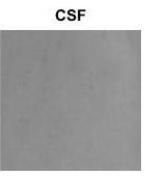

ICSF/case $2 \mathrm{~T} / 0.2$

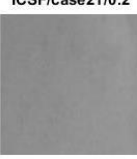

ICSF/case $3 \mathrm{~T} / 0.4$

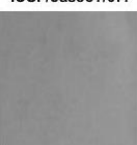

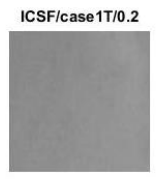

ICSF/case $2 \mathrm{~T} / 0.4$

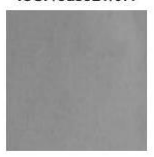

ICSF/case 3 T/0.6

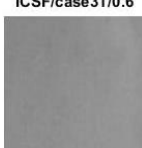

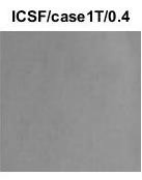

ICSF/case $2 \mathrm{~T} / 0.6$

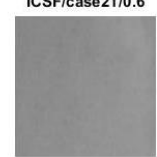

ICSF/case 3 T/ $/ 0.8$

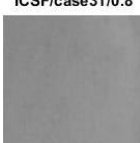

Figure 5. Transformer oil (300C) Gray, OSF, CSF and ICSF images with case 1 , case 2 and case 3 using $\mathrm{C}=0.2,0.4,0.6$ \& 0.8

Figure 5 shows the gray image along with OSF, CSF and ICSF (selecting $\mathrm{C}=0.2,0.4,0.6$ and 0.8 in case 1 , case 2 , case 3 ) filtered transformer oil images. It can be seen that for case $2 / \mathrm{C}=0.2$, the proposed ICSF filter relent improved smoothening of image compared to other filtering methods. The trivial variation of results accomplished in the other cases of ICSF for all the values of $\mathrm{C}$ along with increased values of $\mathrm{C}$ in the same case.

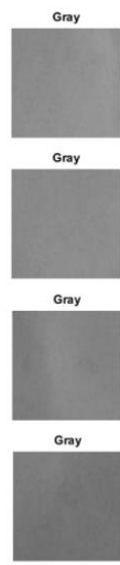

(a)

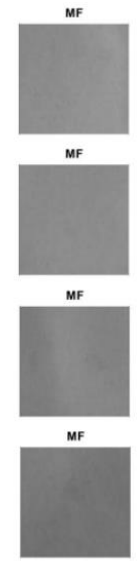

(b)

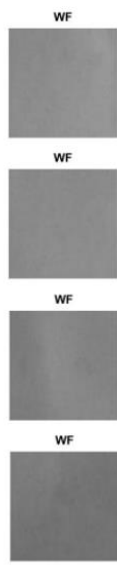

(c)

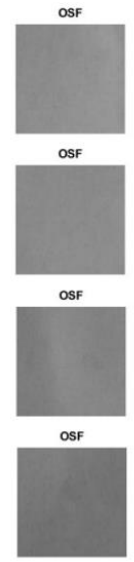

(d)

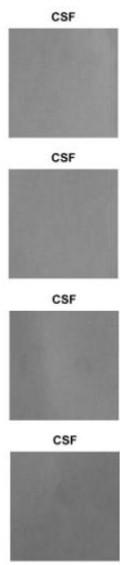

(e)

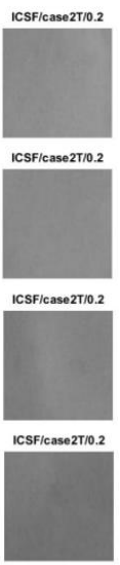

(f)
Figure 6. Top to bottom gray images of transformer oil at $30{ }^{\circ} \mathrm{C}, 60{ }^{\circ} \mathrm{C}, 90^{\circ} \mathrm{C}$ and at $120^{\circ} \mathrm{C}$ temperatures. Enhanced images using (b) Median Filter, (c) wiener filter, (d) OSF, (e) $\mathrm{CSF}$, and (f) ICSF/CASE2/C $=0.2$

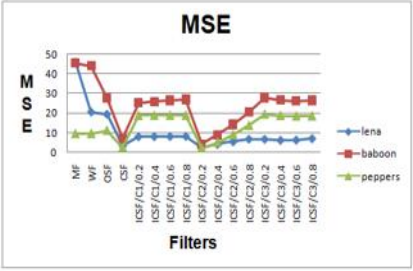

(a)

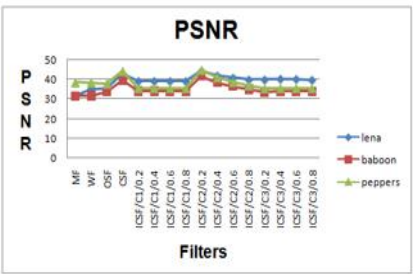

(c)

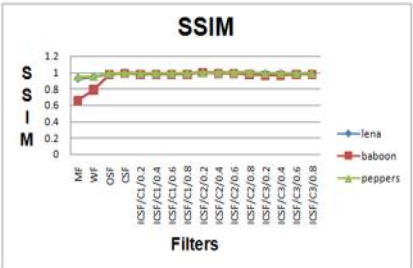

(e)

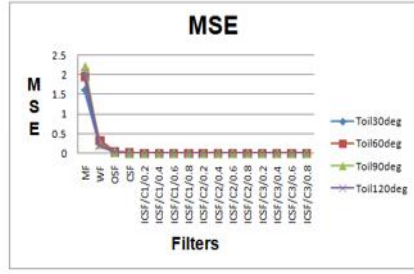

(b)

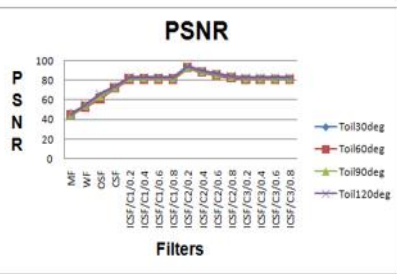

(d)

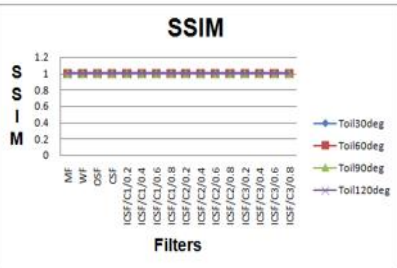

(f)
Figure 7. (a), (c) and (e) MSE, PSNR and SSIM for classical images (b), (d) and (f) MSE, PSNR and SSIM for transformer oil images

\subsection{Performance on transformer oil images}

Further, the performance of the proposed ICSF method tested on transformer oil images and comparison of results are made with the median, wiener, shock and complex shock filtering methods. To denoise, real scalar correction constant $\mathrm{C}$ introduced in proposed ICSF method whose values ranges between $0.1-0.8$. In this paper, $\mathrm{C}$ values of $0.2,0.4,0.6$ and 0.8 are considered in all the three cases (Eq. (5) to Eq. (7)). 
Table 1. Summarizing image quality metrics (MSE, PSNR \& SSIM) of different filters

\begin{tabular}{|c|c|c|c|c|c|c|c|c|c|}
\hline \multirow{2}{*}{\multicolumn{2}{|c|}{$\begin{array}{c}\text { Image } \\
\text { Filtering Method }\end{array}$}} & \multirow{2}{*}{$\begin{array}{c}\text { Image } \\
\text { Quality } \\
\text { Assessment } \\
\text { Metrics }\end{array}$} & \multicolumn{7}{|c|}{ IMAGES } \\
\hline & & & Lena & Baboon & peppers & $\begin{array}{c}\text { Transformer } \\
\text { oil }\left(3^{\circ} \mathbf{C}\right)\end{array}$ & $\begin{array}{c}\text { Transformer } \\
\text { oil }\left(60^{\circ} \mathrm{C}\right)\end{array}$ & $\begin{array}{l}\text { Transformer } \\
\text { oil }\left(90^{\circ} \mathrm{C}\right)\end{array}$ & $\begin{array}{c}\text { Transformer } \\
\text { oil }\left(120^{\circ} \mathrm{C}\right)\end{array}$ \\
\hline \multirow{3}{*}{\multicolumn{2}{|c|}{ Median Filter }} & MSE & 45.96 & 45.33 & 9.45 & 1.61 & 1.94 & 2.19 & 2.04 \\
\hline & & PSNR & 31.542 & 31.603 & 38.411 & 46.106 & 45.288 & 44.762 & 45.069 \\
\hline & & SSIM & 0.93 & 0.66 & 0.95 & 1.00 & 1.00 & 1.00 & 1.00 \\
\hline \multirow{3}{*}{\multicolumn{2}{|c|}{ Weiner Filter }} & MSE & 20.63 & 44.04 & 9.53 & 0.26 & 0.33 & 0.23 & 0.21 \\
\hline & & PSNR & 35.020 & 31.726 & 38.372 & 53.942 & 53.046 & 54.606 & 54.960 \\
\hline & & SSIM & 0.95 & 0.79 & 0.95 & 1.00 & 1.00 & 1.00 & 1.00 \\
\hline \multirow{3}{*}{\multicolumn{2}{|c|}{ Shock Filter }} & MSE & 19.57 & 27.61 & 11.12 & 0.03 & 0.05 & 0.03 & 0.02 \\
\hline & & PSNR & 35.250 & 33.754 & 37.705 & 63.522 & 61.453 & 63.903 & 65.970 \\
\hline & & SSIM & 0.98 & 0.98 & 0.99 & 1.00 & 1.00 & 1.00 & 1.00 \\
\hline \multirow{4}{*}{\multicolumn{2}{|c|}{ Complex Shock Filter }} & MSE & 3.33 & 7.13 & 2.47 & 0.00334 & 0.004 & 0.0030 & 0.0028 \\
\hline & & PSNR & 42.93 & 39.636 & 44.236 & 72.92 & 72.06 & 73.355 & 73.660 \\
\hline & & SSIM & 1.00 & 0.99 & 0.99 & 1.00 & 1.00 & 1.00 & 1.00 \\
\hline & & MSE & 8.12 & 25.30 & 18.68 & 0.00042196 & 0.00049685 & 0.00035656 & 0.00034390 \\
\hline \multirow{2}{*}{$\begin{array}{l}\text { Improved } \\
\text { Complex }\end{array}$} & Case 1 & PSNR & 39.064 & 34.131 & 35.44 & 81.9120 & 81.2024 & 82.6434 & 82.8003 \\
\hline & & SSIM & 0.99 & 0.98 & 0.99 & 1.00 & 1.00 & 1.00 & 1.00 \\
\hline \multirow{7}{*}{$\begin{array}{l}\text { Shock Filter } \\
\text { (scalar } \\
\text { correction } \\
\text { constant } \\
\mathbf{C = 0 . 2 )}\end{array}$} & & MSE & 2.46 & 4.38 & 2.16 & 0.00003087 & 0.00003691 & 0.00002725 & 0.00002573 \\
\hline & Case 2 & PSNR & 44.257 & 41.749 & 44.80 & 93.26937 & 92.4936 & 93.8105 & 94.0601 \\
\hline & & SSIM & 1.00 & 1.00 & 1.00 & 1.00 & 1.00 & 1.00 & 1.00 \\
\hline & & MSE & 6.821 & 27.98 & 19.332 & 0.00046481 & 0.00054842 & 0.00039633 & 0.00038066 \\
\hline & Case 3 & PSNR & 39.826 & 33.694 & 35.301 & 81.4919 & 80.7736 & 82.1841 & 82.3593 \\
\hline & & SSIM & 0.99 & 0.97 & 0.99 & 1.00 & 1.00 & 1.00 & 1.00 \\
\hline & & MSE & 8.17 & 25.84 & 18.713 & 0.00043209 & 0.00050902 & 0.00036596 & 0.00035260 \\
\hline \multirow{2}{*}{$\begin{array}{l}\text { Improved } \\
\text { Complex }\end{array}$} & Case 1 & PSNR & 39.04 & 34.040 & 35.443 & 81.8089 & 81.0974 & 82.5303 & 82.6919 \\
\hline & & SSIM & 0.99 & 0.98 & 0.99 & 1.00 & 1.00 & 1.00 & 1.00 \\
\hline \multirow{7}{*}{$\begin{array}{l}\text { Shock Filter } \\
\text { (scalar } \\
\text { correction } \\
\text { constant } \\
\mathbf{C}=\mathbf{0 . 4})\end{array}$} & & MSE & 4.22 & 9.094 & 5.17 & 0.00008989 & 0.00010656 & 0.00007782 & 0.00007420 \\
\hline & Case 2 & PSNR & 41.90 & 38.577 & 41.02 & 88.6275 & 87.8886 & 89.2537 & 89.4606 \\
\hline & & SSIM & 1.00 & 0.99 & 1.00 & 1.00 & 1.00 & 1.00 & 1.00 \\
\hline & & MSE & 6.28 & 26.688 & 18.61 & 0.00046481 & 0.00054842 & 0.00039633 & 0.00038066 \\
\hline & Case 3 & PSNR & 40.180 & 33.901 & 35.466 & 81.4919 & 80.7736 & 82.1841 & 82.3593 \\
\hline & & SSIM & 0.99 & 0.97 & 0.99 & 1.00 & 1.00 & 1.00 & 1.00 \\
\hline & & MSE & 8.22 & 26.42 & 18.75 & 0.00044261 & 0.00052166 & 0.00037573 & 0.00036163 \\
\hline \multirow{2}{*}{$\begin{array}{c}\text { Improved } \\
\text { Complex }\end{array}$} & Case 1 & PSNR & 39.010 & 33.94 & 35.43 & 81.7044 & 80.9908 & 82.4159 & 82.5821 \\
\hline & & SSIM & 0.99 & 0.98 & 0.99 & 1.00 & 1.00 & 1.00 & 1.00 \\
\hline \multirow{7}{*}{$\begin{array}{c}\text { Shock Filter } \\
\text { (scalar } \\
\text { correction } \\
\text { constant } \\
\text { C = 0.6) }\end{array}$} & & MSE & 5.54 & 14.39 & 9.01 & 0.00018189 & 0.00021503 & 0.00015619 & 0.00014951 \\
\hline & Case 2 & PSNR & 40.727 & 36.583 & 38.612 & 85.5667 & 84.8396 & 86.2282 & 86.4179 \\
\hline & & SSIM & 1.00 & 0.99 & 1.00 & 1.00 & 1.00 & 1.00 & 1.00 \\
\hline & & MSE & 6.42 & 26.22 & 18.338 & 0.00046481 & 0.00054842 & 0.00039633 & 0.00038066 \\
\hline & Case 3 & PSNR & 40.086 & 33.977 & 35.531 & 81.4919 & 80.7736 & 82.1841 & 82.3593 \\
\hline & & SSIM & 0.99 & 0.98 & 0.99 & 1.00 & 1.00 & 1.00 & 1.00 \\
\hline & & MSE & 8.29 & 27.003 & 18.786 & 0.00045352 & 0.00053480 & 0.00038585 & 0.00037098 \\
\hline \multirow{2}{*}{$\begin{array}{l}\text { Improved } \\
\text { Complex }\end{array}$} & Case 1 & PSNR & 39.003 & 33.850 & 35.42 & 81.5987 & 80.8828 & 82.3005 & 82.4712 \\
\hline & & SSIM & 0.99 & 0.98 & 0.99 & 1.00 & 1.00 & 1.00 & 1.00 \\
\hline \multirow{6}{*}{$\begin{array}{l}\text { Shock Filter } \\
\text { (scalar } \\
\text { correction } \\
\text { constant } \\
\mathbf{C}=\mathbf{0 . 8})\end{array}$} & & MSE & 6.88 & 20.552 & 13.60 & 0.00030686 & 0.00036232 & 0.00026236 & 0.00025167 \\
\hline & Case 2 & PSNR & 39.784 & 35.036 & 36.828 & 83.2952 & 82.5738 & 83.9757 & 84.1564 \\
\hline & & SSIM & 0.99 & 0.98 & 1.00 & 1.00 & 1.00 & 1.00 & 1.00 \\
\hline & & MSE & 7.15 & 26.575 & 18.428 & 0.00046481 & 0.00054842 & 0.00039633 & 0.00038066 \\
\hline & Case 3 & PSNR & 39.622 & 33.919 & 35.509 & 81.4919 & 80.7736 & 82.1841 & 82.3593 \\
\hline & & SSIM & 0.99 & 0.98 & 0.99 & 1.00 & 1.00 & 1.00 & 1.00 \\
\hline
\end{tabular}

Figure 6 illustrate OSF, $\mathrm{CSF}$ and $\mathrm{ICSF} /$ case $2 / \mathrm{C}=0.2$ images in addition to gray transformer oil images at $30{ }^{\circ} \mathrm{C}$, $60{ }^{\circ} \mathrm{C}, 90^{\circ} \mathrm{C}$ and $120^{\circ} \mathrm{C}$ temperatures respectively. In order to quantify the performance of each filter, MSE, PSNR and SSIM are determined. A better filter should have low MSE, high PSNR and about to unity SSIM. The calculated values of MSE, PSNR and SSIM for transformer oil image at different temperature using each filter are tabularize in the last four columns of Table 1 . It can be observed from Table 1 that the values of MSE, PSNR and SSIM are low, high and unity respectively in case 2 with $\mathrm{C}=0.2$ of proposed ICSF filter.

\section{CONCLUSIONS}

This paper presents a novel ICSF method for transformer oil images which are captured at different temperatures. This method involves three different cases i.e., each term of CSF equation multiplied with real scalar constant to evolve three different cases of ICSF method. Experiments were performed on synthetic as well as real transformer oil images. The visual as well as numerical results of ICSF method of all three cases are checked for all the images. ICSF method furnish superior results for all the images in case 2 with $\mathrm{C}=0.2$. The results of all the images are validated using image performance quality metrics such as MSE, PSNR and SSIM. The visual as well as numerical results shows ICSF method provide better 
performance when compared with median, wiener, OSF in addition to CSF filter. Finally, this paper concludes the proposed filtering method performance is optimal compared to other classical state of the art filtering methods. Further this proposed filter can be used for segmentation, feature extraction of an image.
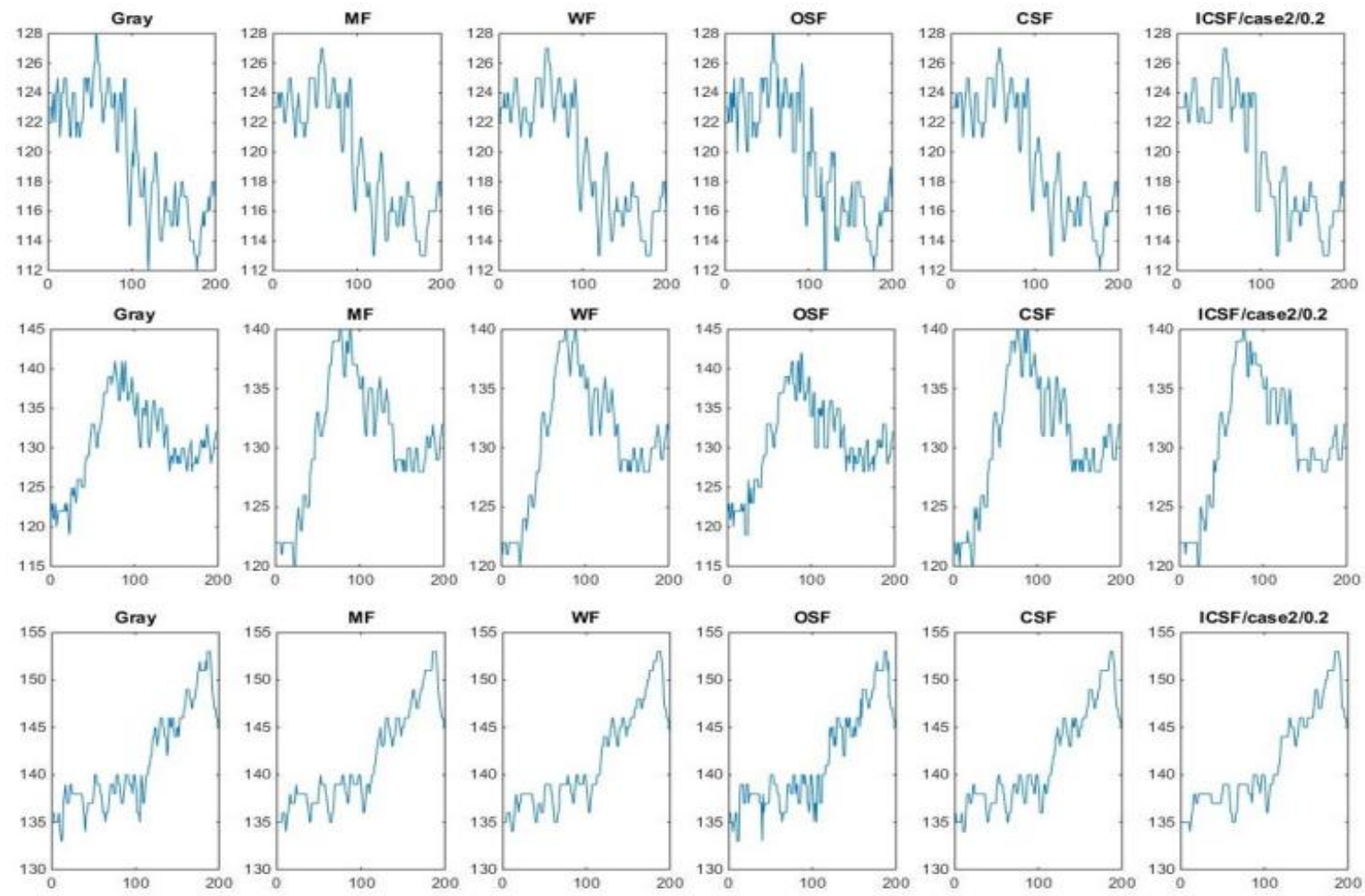

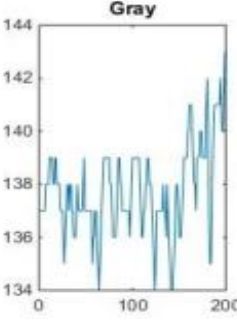

(a)

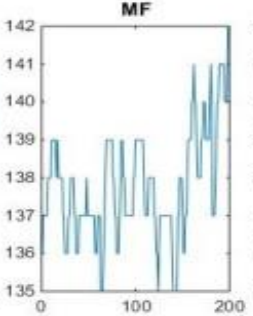

(b)

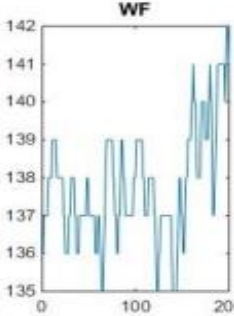

(c)

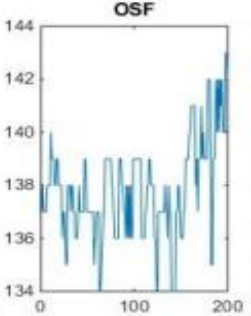

(d)

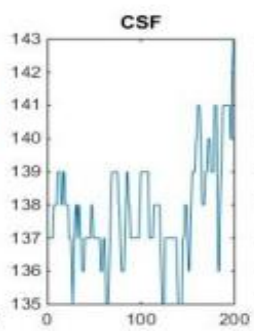

(e)

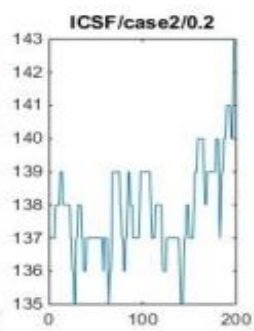

(f)

Figure 8. (a) Top to bottom one dimensional images (gray) of transformer oil at $30{ }^{\circ} \mathrm{C}, 60{ }^{\circ} \mathrm{C}, 90{ }^{\circ} \mathrm{C}, 120^{\circ} \mathrm{C}$. Enhanced images using (b) Median Filter, wiener filter, (d) OSF, (e) CSF, and (f) ICSF/CASE2/C $=0.2$

\section{REFERENCES}

[1] Rouse, T.O. (1998). Mineral insulating oils in transformers. DEIS Feature Article, IEEE Electrical Insulation Magazine, 14: 6-16. https://doi.org/10.1109/57.675572

[2] Martin, J., Heathcote, J.P. (1998). Transformer Book: A Practical Technology of the Power Transformer. Twelfth edition. ISBN 075061158 8. A division of Reed Educational and Professional Publishing Ltd.

[3] Harlow, J.H. (2004). Electric Power Transformer Engineering. CRC Press.

[4] Wang, H., Butler, K.L. (2002). Modeling transformers with internal incipient faults. IEEE Transactions on Power Delivery, 17(2): 500-509. https://doi.org/10.1109/61.997926

[5] Ciulavu, C., Helerea, E. (2008). Power transformer incipient faults monitoring. Annals of the University of Craiova, Electrical Engineering series, (32): 72-77.

[6] Sun, H.C., Huang, Y.C., Huang, C.M. (2012). A review of dissolved gas analysis in power transformers. Energy
Procedia 14, Elsevier Ltd, 14: 1220-1225, https://doi.org/10.1016/j.egypro.2011.12.1079

[7] Maheshan, C.M., Kumar, H.P. (2018). A review of image processing applications in fault detection of transformer oil.

ICTTSTM, https://doi.org/16.10089.IJSRR.2018.V7I06.5245.2445

[8] Gonzalez, R.C., Woods, R.E. (2002). Digital Image Processing. Addison-Wesley Publishing Company.

[9] Jayaraman, S., Esakkirajan, S., Veerakumar, T. (2011). Digital Image Processing. Tata McGraw Hill, ISBN: 9780070144798 0070144796. OCLC Number: 635355917.

[10] Waychal, A.S., Bhosale, Y.N., Kulkarni, S. (2013). Analysis of transformer oil by using MATLAB (image processing tool). International Conference on Energy Efficient Technologies for Sustainability, pp. 11441148. https://doi.org/10.1109/iceets.2013.6533547

[11] Kumar, H.P., Srinivasan, S. (2013). Despeckling of polycystic ovary ultrasound images by fuzzy filter. Recent Advancements in System Modelling Applications. Lecture Notes in Electrical Engineering, 
188: 443-450. https://doi.org/10.1007/978-81-3221035-1_39

[12] Kramer, H.P., Bruckner, J.B. (1975). Iterations of a non linear transformation for enhancement of digital images. Patteren Recognition, 7(1-2): 53-58 https://doi.org/10.1016/0031-3203(75)90013-8

[13] Osher, S., Rudin, L.I. (1990). Feature-oriented image enhancement using shock filters. SIAM Journal on Numerical Analysis, 27: 919-940. https://doi.org/10.1137/0727053

[14] Alvarez, L., Mazorra, L. (1994). Signal and image restoration using shock filters and anisotropic diffusion. SIAM Journal on Numerical Analysis, 31(2): 590-605. https://doi.org/10.1137/0731032

[15] Gilboa, G., Sochen, N.A., Zeevi, Y.Y. (2004). Image enhancement and denoising by complex diffusion processes. IEEE Transactions on Pattern Analysis and Machine Inlelligence, 26(8): 1020-1036. https://doi.org/10.1109/tpami.2004.47

[16] Kudelka Jr, M. (2012). Image Quality Assessment. WDS'12 Proceedings of Contributed Papers, Part I, pp 94-99. ISBN 978-80-7378-224-5 (c) MATFYZPRESS.

[17] Alain, H., Djemel, Z. (2010). Image quality metrics: PSNR vs. SSIM. International Conference on Pattern Recognition, pp. 2366-2369. https://doi.org/10.1109/ICPR.2010.579

[18] Sara, U., Akter, M., Uddin, M.S. (2019). Image quality assessment through FSIM, SSIM, MSE and PSNR-A comparative study. Journal of Computer and Communications, $\quad 7$ : $\quad 8-18$. https://doi.org/10.4236/jcc.2019.73002

[19] Dung, L.R., Sun, S.T., Wu, Y.Y. (2014). Implementation of shock filter for digital $\mathrm{X}$-ray image processing. Journal of Computer and Communications, 2(13): 25-33. http://dx.doi.org/10.4236/jcc.2014.213004. 